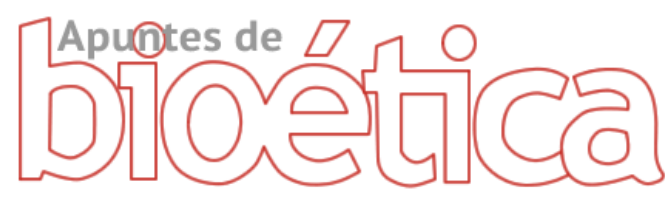

https://doi.org/10.35383/apuntes.v2i1.242

\title{
Enseñanza de la Bioética en la Educación Universitaria
}

\author{
León Llacsa Soto ${ }^{1}$
}

\section{INFORMACIÓN DEL ARTÍCULO RESUMEN}

Historia del artículo:

Recibido el 21 de marzo de 2019

Aceptado el 26 de julio de 2019

Palabras claves:

Bioética

Ética

Educación universitaria

Se revisa el tema, considerado al inicio como ética, y su realidad académica en la Universidad Se fundamenta la necesidad de la enseñanza de la bioética, su desarrollo, en todas las profesiones y no sólo en salud. Que su objetivo, es generar conocimientos, aprendizajes, reflexiones, actitudes y comportamiento ético sociales. En su metodología resalta el aprendizaje basado en problemas, por favorecer el razonamiento ético, cambio de actitudes y trabajo en equipo; los Seminarios y los estudios de caso como más usados. Se concluye que en la universidad es imprescindible la formación en bioética para mejorar la calidad educativa, promover comportamientos éticos con sentido, personal, profesional y social; que el aprendizaje basado en problemas, seminarios, estudio de casos, entre otros, permiten aprendizajes que respetan, valores, derechos, democracia y ambiente. Así mismo que la responsabilidad social de la calidad educativa es función de la gestión institucional y de sus actores.

\section{Bioethics teaching in university education}

\section{ABSTRACT}

\section{Keywords:}

Bioethics

Ethics

University education
It is based on the need for the teaching of bioethics, its development, in all professions and not only in health. That its objective is to generate knowledge, learning, reflections, attitudes and social ethical behavior. Its methodology emphasizes problem-based learning, favoring ethical reasoning, changing attitudes and teamwork; Seminars and case studies as the most used. It is concluded that at the university, training in bioethics is essential to improve educational quality, promote ethical behaviors with meaning, personal, professional and social; that learning based on problems, seminars, case studies, among others, allow learning that respects values, rights, democracy and environment. Likewise, social responsibility for the quality of education is a function of institutional management and its actors.

\footnotetext{
${ }^{1}$ Médico Cirujano Gineco-Obstetra, Catedrático Principal de la Facultad de Medicina Humana de la Universidad Nacional San Luis Gonzaga, Magister en Educación con Mención en Planificación Educativa - UNSL, Miembro del Comité de Ética del Consejo Regional Colegio Médico del Perú. Lima, Perú. Email: leonllacsa7@gmail.com.ORCID: https://orcid.org/0000-0002-9667-5664
} 


\section{Introducción}

La ética fundamento de la moral, se impartió en la Universidad desde su creación dentro de la filosofía, posteriormente dejada de lado en los años de la reforma universitaria, y en la década de los 70 a 90 del siglo anterior se reincorpora en las ramas de medicina y derecho, e incluye los fundamentos de la ética, moral, el aprendizaje de normas basadas en principios, valores y virtudes, más tarde las normas deontológicas en los códigos de ética profesional orientados al cumplimiento de sus deberes como profesionales ante la sociedad.

Las universidades pioneras en enseñar ética son: Harvard, Columbia, Maryland, Pensilvania, Barcelona, Pontificia de Madrid y Valencia, más tarde en América latina: Alberto Hurtado de Chile, Rosario de Colombia, Autónoma de México, Sao Paulo Brasil y Córdova Argentina (Pérez \& Fernández ).

La bioética tiene un discurso científico, dialógico, secular, procedimental e interdisciplinar aún, que no todos lo comprenden e interpretan como tal, lo que realmente determina es que amplía totalmente el contexto de la ética tradicional, del plano predominante personal y deontológico a un plano colectivo y también social cultural y ambiental.

Son muchos aún quienes confunden ética con moral, deontología con moralidad, y la misma ética con la bioética y no se hace el esfuerzo por comprender e interiorizar el propósito real de la bioética: que es el de contribuir a una racionalidad emancipadora en el marco del diálogo plural, interdisciplinario, democrático y respetuoso de las diferencias en convivencia (Outomuro, 2008).

Las instituciones fundamentalmente educativas, son como espacios abiertos al mundo para reinterpretarlo y reconocer a través de este y donde se propicie la explicación, al desarrollo y a la orientación de las virtudes y potencialidades del individuo como persona $y$ futuro ciudadano, al decir de Savater "la verdadera educación no consiste sólo en enseñar a pensar, sino también en aprender a pensar sobre lo que se piensa".

Deben constituirse luego en ámbitos de desarrollo del entendimiento y la convicción por la autonomía y la responsabilidad, de formar conciencias orientadas a la autotrascendencia y a la autenticidad, por la elección de valores que aseguren la supervivencia de la humanidad, en un proceso ascendente hacia su plena realización evolutiva.

En el ámbito mundial actual predomina un modelo económico y político que no respeta la dignidad humana y a sus colectividades, a pesar de estar establecido y legislado a nivel internacional, el neoliberalismo propicia la desigualdad y la minimización de las funciones económica y social de los estados.

Ante ello la humanidad tiene la necesidad urgente de encontrar perspectivas de sobrevivencia o mediación a través de un diálogo entre la ciencia y las humanidades o entre el conocimiento científico y los valores humanos (Bermúdez, 2006).

La Universidad como espacio de encuentro de saberes es el lugar más indicado para reflexionar y aprender desde la bioética sobre los problemas de la interacción: vida, ciencia, tecnología, sociedad e investigación, para aportar perspectivas ético-morales, al desarrollo y evolución de la humanidad (Rodriguez, 2007).

\footnotetext{
${ }^{2}$ Lo cita en su obra "El valor de educar". Barcelona; Ariel; 1997:32.
} 
Un estudio para identificar la bioética en los planes de estudio de 76 facultades de medicina calificadas de Brasil en el 2010 reveló que en 10 de ellas no existía, en el $47 \%$ como bioética, en el $38 \%$ como bioética asociada a otras disciplinas, y en $15 \%$ asociadas.

Que, si bien hubo aumento de la disciplina como bioética, de sus contenidos a otras disciplinas y predominio de vínculo a la ética profesional y deontología, es necesario su mayor desarrollo y fortalecimiento como disciplina académica de pregrado y como básica para los cursos de especialización y posgrado (Neves, Serpa, \& Rego, 2016).

\section{Objetivo}

El objetivo general es, el de contribuir a la formación del tipo de profesional que la sociedad necesita, los egresados deben poseer las características esenciales de cada profesión, pero también conocer las características específicas de su entorno cultural, cívico y social. Se señalan: primero, entregar y generar conocimientos en una visión interdisciplinar; segundo, promover el cambio de actitudes y de comportamientos; tercero, trasmitir los valores éticos más apropiados y necesarios para todo profesional.

La participación del docente es fundamental y exige permanente actualización, si bien se forma en una disciplina científica determinada, está obligado a formarse una visión multidisciplinar y a capacitarse en ética, deontología, bioética y los profesores titulares con los diplomados y maestrías que ya existen.

El campo de la bioética se fue ampliando cada vez más desde la ética aplicada, clínica o particular a una ética global y ambiental y a los problemas de las éticas institucionales y de los sistemas de salud, judicial, educativo, económico, etc. hay propuestas respecto a los contenidos, como el de Abel. $\mathrm{F}^{3}$, por el que los objetivos docentes se deben orientar en torno a tres ejes:

Desarrollo progresivo de la competencia profesional, como ejercicio en la construcción de su personalidad moral.

Armonización entre los valores del conocimiento científico y los valores del conocimiento global y humanístico de las personas.

La sinergia entre los objetivos y los medios o recursos para llevarla a buen término (Francisco, 2008).

Existe aún debate sobre la mejor manera de enseñar la ética y/o la bioética o si es posible impartirlas en el ámbito de la educación universitaria en general. Así mismo hay aún real desinterés por parte de quienes gestionan las instituciones educativas sobre lo que se puede o se debe hacer en términos de educación ética.

En la actualidad no es coherente entender la bioética basada sólo en códigos deontológicos que impone más normas que principios, es necesario volver a la reflexión filosófica, deliberar y tomar decisiones para que se eduque moralmente por el ejemplo en la labor diaria, de evaluar cómo la llevamos a cabo, para mejorar el sentido ético y social de las acciones y así detectar los aspectos positivos o negativos.

Aprender a descubrir valores que se lleva o adquiere, aplicarlos con responsabilidad y valor para sostenerlos en la deliberación con mente abierta, respeto y prudencia hasta la toma de decisiones, en un modelo educador que desarrolle humanidad (Vidal, 2012).

Se enfatiza que el objetivo principal de la enseñanza de la bioética debe ser: desarrollar en

\footnotetext{
${ }^{3}$ Descrito en su artículo: "Diálogo interdisciplinar". Cuadernos de Bioética, 1999;37(1):11-16
} 
los estudiantes el proceso de búsqueda de soluciones a través de múltiples habilidades, principalmente el de la capacidad de juicio ético moral, definida como la capacidad de tomar decisiones y hacer juicios morales sobre la base de principios y valores internos, para identificar y discernir sus intereses morales individuales y los colectivos.

Así mismo que la bioética debe cumplir doble función: 1) ayudar al estudiante en el desarrollo de su carácter moral, actitudes y su capacidad de análisis bioético que incluye la deliberación con toma de decisiones y 2) formar a ciudadanos comprometidos con el bien común (Neves, Serpa, \& Rego, 2016).

La UNESCO a propósito de la necesidad de la enseñanza de la bioética, expresa que corresponde a todos los Estados enseñarla ante los nuevos problemas que enfrenta la humanidad respecto a los altos avances de la ciencia y la tecnología, estableciendo a través del Artículo 23 de la Declaración de Bioética y Derechos Humanos (2005) un programa de educación permanente de bioética, con los propósitos de:

Una nueva concepción del sujeto: racionales y libres, reflexivos y críticos, creadores y transformadores de sus prácticas.

Contextualizada en base a las prácticas de los sujetos.

Problematizadora, interroga y construye problemas sobre el contexto y las prácticas a través del diálogo intersubjetivo.

Continua: dura toda la vida.

Para el cambio de actitudes primarias y secundarias (Torres).

Se considera en su análisis respecto a una óptima educación que ésta disciplina, la bioética debe incluirse y enseñarse en los aspectos propios de la vida, el trabajo, la convivencia y el respeto a sí mismo, a los demás y a su ambiente, en un mundo enmarcado por el paradigma de la globalización, el mercado y la interdependencia, orientada a mejorar la calidad de las acciones hacia una sociedad solidaria, participante $y$ creativa.

\section{Metodología}

Dentro del modelo educativo del constructivismo y la concepción de un currículo basado en competencias se reporta que la metodología educativa más aceptada en el nivel universitario desde la década de los noventa y en aplicación actual en buen número de países, es la del aprendizaje basado en problemas (ABP) (Couceiro, 2008).

Ubicada dentro de la concepción centrada en el estudiante, el aprendizaje significativo y por descubrimiento, el razonamiento crítico que busca adquirir conocimientos, desarrollar habilidades y actitudes a partir de problemas, estimular al trabajo en equipo y el saber escuchar opiniones, así como también facilita el razonamiento ético y la toma de decisiones (Chávez).

Entre los principales efectos del ABP sobre el aprendizaje se destacan:

- Facilita la comprensión de los nuevos conocimientos

- Promueve la motivación en los actores

- Provoca conflictos cognitivos

- El aprendizaje resulta de la cooperación y colaboración

- Permite la actualización de la zona de desarrollo próximo de los estudiantes (Iturria, 2007).

En una sociedad tecnocientífica, multicultural y en debate de pertinencia de juicios críticos y razonables ante el manejo de recursos naturales, impacto ambiental de 
desechos industriales, contaminación, biodiversidad, aspectos sociales como la pobreza y la efectividad de las políticas sectoriales de los gobiernos (Ovalle, Escobar, \& Aristizábal, 2010).

Se admite que el proceso formativo en bioética es posible y debería transformar actitudes y carácter moral de las personas, aunque muchos no lo crean es, más posible también que pudiera darse una transformación negativa, porque la experiencia humana $y$ docente puede no sólo potenciar, sino inhibir su desarrollo moral, al percibir clara distancia entre lo que se les comunica como valores y los ejemplos que observan, lo que constituye un reto al docente y a la institución educativa a que estimule continuamente al desarrollo moral positivo (León, 2014).

En la metodología universitaria vigente los Seminarios es la principal técnica grupal de aprendizaje activo, es aplicado en la enseñanza de la ética y en todas las áreas del conocimiento, como estudio selectivo de temas en sesiones planificadas usando fuentes de información autorizadas, con mayor participación, trabajo de investigación en equipo y labor colaborativa mutua y con el maestro.

Otro método es el estudio de casos, que en medicina se desarrolla bastante como análisis de casos ético-clínicos, al que se integra la bioética con principios, valores y aún con sus diferentes corrientes, para alcanzar luego del análisis, deliberación y consenso, el objetivo común de resolver o dar alternativas en la resolución de los casos, y que también es aplicable a diferentes profesiones.

Se promueve el método integrado para el análisis de casos presentados como dilemas, toma en cuenta los cuatro principios éticos y las circunstancias del caso. El método comprende: una primera fase de desarrollo de una sensibilidad ética, una segunda fase de información, análisis, posibles cursos de acción, toma de decisiones y la ejecución. El análisis de los posibles cursos de acción es el punto crucial que, junto a la toma de decisiones, ponen a prueba la formación que se tenga en bioética, destaca la aplicación continua de la prudencia que da unidad al análisis y profundiza la reflexión al aplicar principios, juicios y decisiones hasta en la implementación práctica de la solución propuesta (Arratia, Casas, \& Rodriguez, 2010).

Entre otros métodos que se mencionan está el de las lecturas y comentarios de texto que se usan en ciencias sociales y de humanidades, en relación próxima se encuentra también las monografías no descriptivas temáticas sino como ensayos críticos de análisis e interpretación deductiva personal.

Como actividades de autoaprendizaje, están considerados el uso de los instrumentos pedagógicos electrónicos que facilitan el acceso a las herramientas virtuales de comunicación: foros, videos, blogs, software y uso de las bases de datos que complementan con eficacia el proceso formativo de los usuarios (Vera, 2012).

El uso de las dramatizaciones o juego de roles o performance actoral de situaciones a través del diálogo y de la forma oral y gestual ante un público comunitario determinado en las actividades de extensión social, son métodos que considerar y evaluar, para conmover o transmitir contenidos educativos en un marco natural más apropiado donde el educando desarrolle su sentido ético/bioético en una sociedad más incluyente (Cantú, 2015).

\section{Resultados, análisis y discusión}

Se refiere que la enseñanza de la ética en la Universidad desde hace más de dos décadas se basa aún en metodologías tradicionales que no promueven la participación y el diálogo entre los 
estudiantes, con un bajo grado de motivación y conocimientos logrados.

Se reconoce y fundamenta la necesidad imperativa de la enseñanza-aprendizaje de la ética, como bioética en la Universidad, que incluye a la ética, ética médica, deontología y/o ética profesional, no sólo como prioridad de las profesiones de la salud sino de todas ellas sin excepción, por ser uno de sus propósitos la búsqueda, producción, crítica y difusión de los conocimientos y saberes de la bioética en lo académico, en la investigación y en la proyección a la comunidad.

Reconocer la prioridad de su aplicación práctica en los problemas éticos contemporáneos producidos por el desarrollo científico y tecnológico, con impacto en todas las áreas profesionales, el ambiente social, cultural y el ecosistema; y el de contribuir a la construcción de la ciudadanía y del sistema democrático.

Se refiere que a través de ella se busca propiciar y fomentar las capacidades $y$ responsabilidades en las decisiones y acciones personales, profesionales, colectivas y ambientales, que se configure en una política institucional de enseñanza de la bioética como eje transcurricular en los programas de pregrado, diplomados y postgrado (Ovalle, Escobar, \& Aristizábal, 2010).

Como objetivo se propone el de generar conocimientos, aprendizajes y reflexiones con visión interdisciplinaria, promover actitudes y comportamientos con principios y valores, ante los problemas ético-sociales ocasionados por los efectos de los avances científico-técnicos que se suceden incontenibles, y las relaciones interpersonales y sociales.

Que priorice la competitividad, la armonización de valores y la sinergia entre políticas y medios, que permitiría a los egresados tomar decisiones libres y responsables ante situaciones o dilemas acordes a su realidad, con respeto a la diversidad y donde se privilegie la equidad y la justicia social (Cantú, 2015).

Así mismo se oriente a promover el desarrollo humano, por su desarrollo ético moral y de la conciencia social, hacia una conciencia bioética, base de su madurez cívica ciudadana con responsabilidad social, en la familia, las instituciones y su comunidad, ante los efectos de una sociedad de consumo, de mercado y globalización.

El aprendizaje basado en problemas es el método más propuesto, por incrementar la participación y la motivación para facilitar el desarrollo del razonamiento ético, pensamiento crítico, valores, actitudes, habilidades de comunicación, trabajo en equipo y liderazgo.

El estudiante es el principal actor en el compromiso de búsqueda del conocimiento, de las alternativas de solución de los problemas de su realidad y quien debe asumir la responsabilidad de ser consciente de cada etapa del proceso cognitivo de aprender a aprender nuevos conocimientos y que es posible decidir transformar las actitudes y los comportamientos.

De la metodología vigente los Seminarios son el principal medio de aprendizaje activo y el más usado en áreas profesionales y estudios de postgrado, sus características: el de asegurar al estudiante mayor participación, labor colaborativa de trabajo en equipo, buen ejercicio del diálogo, de la crítica, argumentación y conclusiones, en una experiencia de aprendizaje compartida y planificada.

Otro método importante bastante aplicado en medicina y ciencias de la salud es el estudio de casos, y aplicable a todas las profesiones según sus características propias, configuran una metodología diversa. 
Un complemento importante a todos es el uso de medios didácticos diversos que la tecnología de la información, el internet y el grupo creciente de herramientas virtuales: foros, videos, blogs, software proporcionan medios de comunicación positiva al proceso de aprendizaje y enseñanza sostenido. Así mismo las lecturas, ensayos críticos, dramatizaciones son métodos complementarios importantes para considerar.

Un aspecto no considerado en los reportes es el referido a la situación formal de la bioética en la estructura curricular universitarias que defina su ubicación, profesores, cursos, métodos, contenidos y evaluación pertinentes, consensuadas y coordinadas. Dentro de una gestión institucional que priorice a la excelencia como evidencia de calidad y sentido social. Así mismo el establecimiento de programas de capacitación continua en bioética y humanidades e integrados a su capacitación específica para los profesores, equipos de gestión y aún para los miembros de la comunidad universitaria en general.

\section{Conclusiones}

En el marco de la educación formal y principalmente en el nivel de la educación universitaria, se hace imprescindible la institucionalización de la bioética que consolide y oriente la formación profesional de estudiantes y profesores, como una ética práctica automotivada general y específica, que acompañe la mejora de la calidad del proceso educativo y de la formación integral de profesionales.

Su objetivo: generar conocimientos, aprendizajes, juicios de valor, actitudes y comportamientos ético-sociales adecuados en su desarrollo personal y durante su formación para el ejercicio profesional futuro, a nivel personal, institucional y socio ambiental.
El principal método propuesto es el Aprendizaje basado en problemas por el que se facilita y motiva el proceso enseñanza aprendizaje a partir de problemas, para adquirir conocimientos y desarrollar habilidades y actitudes ético-morales con sentido personal y social.

El Seminario es la técnica de trabajo en grupo más utilizado en todas las profesiones, por su característica de facilitar el aprendizaje activo, por la mayor participación del estudiante, en trabajo colaborativo, dialogal, continuo, con argumentación y conclusiones, como una experiencia de aprendizaje grupal.

Los estudios de caso como aprendizaje basado en casos son más usados en las profesiones de salud en las que tiene mayor desarrollo e incluye a la bioética, y son también aplicables a todas las profesiones. El uso de los instrumentos de la tecnología y comunicación virtual de la información son ahora en herramientas pedagógicas imprescindibles.

Las lecturas, ensayos críticos y dramatizaciones más usados en las ciencias sociales y en la educación básica se consideran también buenos y efectivos complementos.

\section{Recomendaciones}

La responsabilidad social por lograr avances en la mejora de la calidad educativa, la detentan quienes ejercen la gestión institucional y también cada uno de los actores del proceso enseñanza aprendizaje.

\section{Bibliografía}

Abel, F. (1993). Diálogo interdisciplinar. En Cuadernos de Bioética (págs. 11-16). 
Arratia, A., Casas, M., \& Rodriguez, E. (2010). Bioética General y Clínica. Santiago de Chile: Interamericana Ciencia y vida.

Bermúdez, C. (2006). Necesidad de la bioética en la educación. En Acta Bioethica (págs. 3639).

Cantú, P. (2015). Bioética y educación superior en México. En Acta Bioethica (págs. 4748). Monterrey: Universidad Autónoma de Nuevo León.

Chávez, O. (s.f.). La bioética y la educación en ciencias de la salud enfocada en problemas. Universidad de Los Andes Mérida.

Couceiro, A. (2008). Enseñanza de la bioética y planes de estudios basados en competencias. Viguera Editores SI.

Francisco, L. (2008). Enseñar bioética: cómo trasmitir conocimientos actitudes $y$ valores. En Acta Bioethica (págs. 12-16).

Iturria, A. (2007). Aprendizaje Basado en problemas. Santillana.

León, G. (2014). Bioética y educación: educación para la bioética.

Neves, W., Serpa, L., \& Rego, S. (2016). La enseñanza de la bioética en las escuelas de medicina en Brasil.

Outomuro, D. (2008). Fundamentación de la enseñanza de la bioética. En Acta Bioethica (págs. 20-23).
Ovalle, C., Escobar, J., \& Aristizábal, C. (2010). Educación en bioética: experiencia de un programa. Revista Colombiana de bioética.

Pérez, F., \& Fernández, A. (s.f.). La ética profesional en la formación universitaria. Rosario, Colombia. Obtenido de www.nodo50.org: https://www.nodo50.org/cubasiglo/congr eso08/conf4_perezf.pdf

Rodriguez, H. (2007). La bioética en la educación universitaria: perspectiva Hermenéutica. Nueva Granada.

Torres, G. (s.f.). Importancia de la institucionalización de la bioética en la educación superior colombiana. Bogotá: Universidad Militar Nueva Granada.

Vera, O. (2012). Bioética: Planes de estudios de la enseñanza basada en competencias en las Facultades de Medicina. La Paz.

Vidal, S. (2012). Nuevas y viejas preguntas en la educación en bioética, en La educación en bioética en América Latina y Caribe. $18-24$ 